\title{
Numerical study of heat and mass transfer in a desalination system
}

\author{
H. EL BAAMRANI*l, L. BAMMOU ${ }^{1}$, A. AHAROUNE ${ }^{l}$, A. BOUKHRIS ${ }^{l}$ \\ 1Thermodynamics and Energetics Laboratory, Faculty of Science, Ibn Zohr University, BP8106, 80006 Agadir, Morocco \\ *Correspondence author. Email: hayatelbaamrani92@gmail.com
}

\begin{abstract}
In today's world the demand for freshwater, to meet the needs of human activities is growing exponentially. As a result, manufacturers are continuing to make progress in the design and production of efficient desalination and cooling units to optimize and reduce the overall cost of production. In this work, we study the numerical study of the evaporation of a thin liquid film dripping by gravity with constant feed rates in a closed rectangular cavity formed by two parallel flat plates. The wall which supports the liquid film is heated by a constant temperature heating, while the other is kept at a constant and uniform temperature to condense the formed vapor. The results obtained show that the heat transfer in the distillation cell is dominated by the latent heat transfer associated with the evaporation. The results also show that the temperature of the film increases slightly for the heating zone and then decreases over most of the plate for the evaporation zone
\end{abstract}




\section{Introduction}

Due to its wide practical applications (desalination, evaporative cooling, cooling of electronic components, ...), the evaporation of a liquid film has been the subject of several works, both numerical and experimental [1-10]. This work concerned both mixed and natural convection flows. In addition, to simulate the heat and mass transfers with evaporation / condensation of a liquid film, several authors have adopted the hypothesis of a liquid film of negligible thickness [1, 3-4]. This hypothesis makes it possible to overcome the resolution of conservation equations in the liquid film. The film is then treated as a boundary condition. The validity of this hypothesis has been investigated by Yan [8-9]. This author has carried out a numerical study of laminar mixed convection with evaporation of a liquid film dripping on the internal walls of a vertical canal. The walls of the channel are uniformly heated isothermally [9] or with a uniform heat flow [8].

The author has conducted two studies in parallel: in one conservation equations have been solved both in liquid phase and gas phase and in the other the hypothesis of the film of negligible thickness has been adopted. By comparing the results of these two studies, the author was able to show that the condition of validity of the film hypothesis of negligible thickness is that the flow rate of the liquid film is low.

The objective of the present work is to study numerically the heat transfer and mass with phase change in a vertical channel formed by two parallel flat plates. One of the plates is humid and maintained at a constant and uniform temperature while the other is adiabatic and dry. The influences of the film input parameters and the temperature heating applied to the wall were studied.

\section{MATERIALS AND METHODS}

The studied configuration is a vertical channel formed by two parallel flat plates of height $\mathrm{H}$ and distant from $\mathrm{b}$ (Fig. 1). Using this device, the evaporation of a thin liquid film which flows on the inner face of the plate is studied. This liquid film enters the channel with a mass flow $\mathrm{D}_{\mathrm{m}}$, and a temperature $T_{0}$. A uniform heat flux density $\mathrm{q}_{\mathrm{w}}$ is applied to the wet wall.

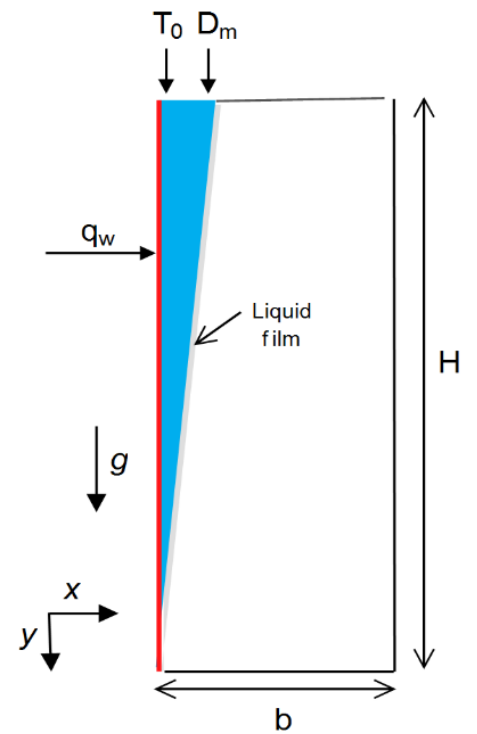

Fig 1. Physical model

Taking into account the abovementioned simplifying hypotheses, the equations governing the heat and mass transfers in a channel are written in the following form:

Continuity equation for VOF model

$$
\frac{\partial}{\partial t}\left(\alpha_{v} \rho_{v}\right)+\nabla \cdot\left(\alpha_{v} \rho_{v} \vec{v}_{v}\right)=\left(\dot{m}_{l v}-\dot{m}_{v l}\right)
$$

The continuity equation solves the secondary phase. The primary phase is calculated using the following equation:

$$
\sum_{p=1}^{n} \alpha_{q}=1
$$

Momentum equation

$$
\frac{\partial}{\partial t}(\rho \vec{v})+\nabla \cdot(\rho \vec{v} \vec{v})=-\nabla_{p}+\nabla \cdot\left[\mu\left(\nabla \vec{v}+\nabla \vec{v}^{T}\right)\right]+\rho \vec{g}+\vec{F}
$$

In (3), $\rho$ and $\mu$ treated as effective values for the combined phase, which are based on the volume fractions of each phase on the following form:

$$
\begin{aligned}
& \rho=\alpha_{l} \rho_{l}+\alpha_{v} \rho_{v} \\
& \mu=\alpha_{l} \mu_{l}+\alpha_{v} \mu_{v}
\end{aligned}
$$


Energy equation

$$
\left.\frac{\partial}{\partial t}(\rho E)+\nabla \cdot(\vec{v}(\rho E+p))=\nabla \cdot\left(K_{e f f} \nabla \mathrm{T}\right)\right]+S_{h}
$$

The VOF model treats the energy as mass averaged variable:

$$
E=\frac{\sum_{p=1}^{n} \alpha_{p} \rho_{p} E_{p}}{\sum_{p=1}^{n} \alpha_{p} \rho_{p}}
$$

The system of equations (1) - (7) is solved numerically by the finite volume method. The mesh is uniform in both directions with a higher density of nodes near the entrance and near the walls where the temperature and concentration gradients are the highest. The calculation code has been validated in comparison with the results of the literature.

\section{Results and discussions}

The results presented here were obtained under the following conditions:

-The problem is two dimensional.

- Ambient air temperature $\mathrm{T}_{0}=25^{\circ} \mathrm{C}$.

-The thermo-physical properties are considered constant.

The simulation results show the temperature and vapor fraction profiles in the liquid film. The shape of the curves describing the evolution of the temperature of the liquid film, figure 2, reveals two zones:

a liquid heating zone situated at the top of the channel, where the temperature exhibits a significant increase as a function of the depth. an area that extends over the rest of the wet surface, where the variation in temperature is minimal. This result is proved in the work of Ben Jabrallah et al. [11].

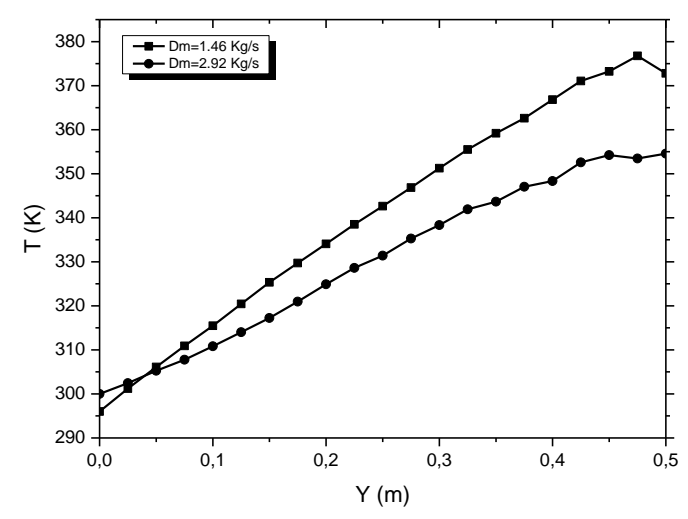

Fig 2. The temperature profiles along the liquid film for $\mathrm{qw}=600 \mathrm{~W} / \mathrm{m} 2$
The temperature is an important parameter that is worth investigating. We represent in Fig. 3, the evolution of the temperature along the liquid film for different heat flux. Firstly, it is noted that the temperature increases along with the heat flux, which is in agreement with most previous works. However, it is obvious that a higher heat flow density resulting a higher temperature along the channel.

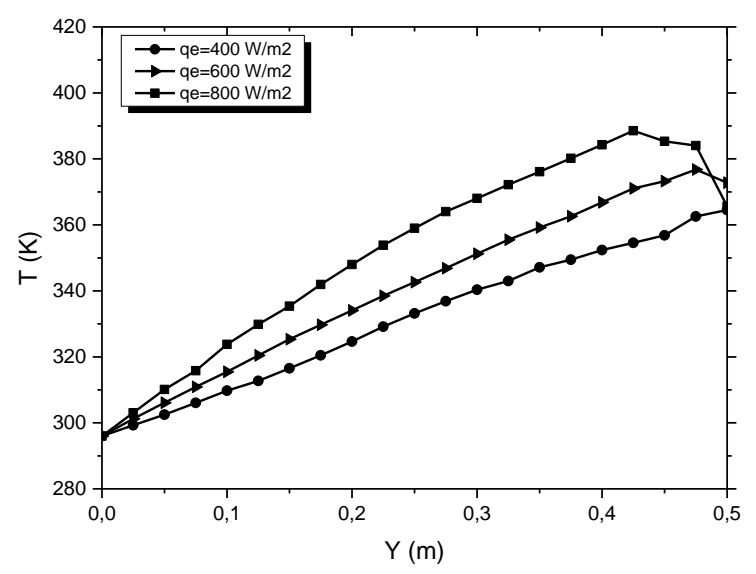

Fig 3. The temperature profiles along the liquid film for $\mathrm{Dm}=1.46 \mathrm{Kg} / \mathrm{s}$

The evolutions of the vapor fraction are presented in Fig. 4 and Fig 5. After one second, the first bubbles of vapors start to be created at liquid film, where the temperature heating was applied. The rise of the vapor bubbles is fast which disturbs the balance of the liquid phase. We also notice that the bubbles begin to form from the bottom of the channel. 


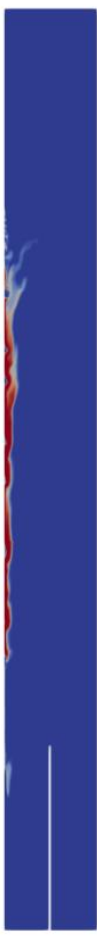

$0.5 \mathrm{~s}$

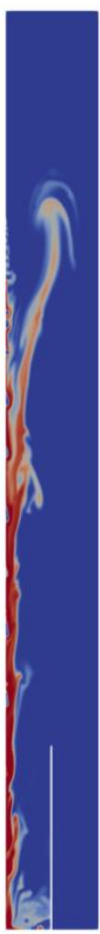

1s

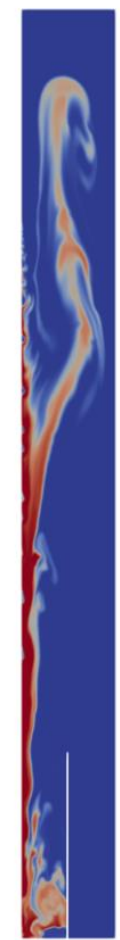

$1.5 \mathrm{~s}$
Fig 4. The vapor fraction contours evolution as a function of time

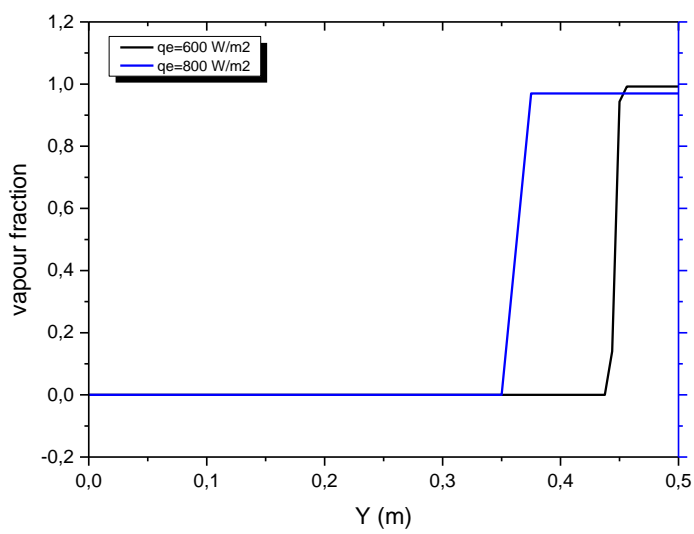

Fig 5. Evolution of the vapour fraction along the channel for $\mathrm{Dm}=1.46 \mathrm{Kg} / \mathrm{s}$

\section{Conclusions}

A numerical study of the evaporation of a film dripping on the heated wall of a distillation cell has been proposed. The influences of the film input parameters and the heating flux applied to the wall were studied. The results obtained show that the temperature of the film increases slightly (heating zone) then decreases over most of the plate (evaporation zone).

\section{References}

[1] Azizi Y., B. Benhamou, N. Galanis and M. El-Ganaoui, Buoyancy Effects On Upward And Downward Laminar Mixed Convection Heat And Mass Transfer in a Vertical Channel, Int. J. Num. Meth. Heat Fluid Flow, 17-3 (2007), 333-353

[2] E. C. Siow, S. J. Ormiston, H. M. Soliman, Two-phase model of laminar film condensation from vapour-gaz mixtures in declining parallel-plate channels, Int. J. of Thermal and Sciences, 46 (2007), 458-466.

[3] Z. Ait Hammou, B. Benhamou, N. Galanis and J. Orfi, Laminar Mixed Convection of humid Air in a vertical channel with evaporation or condensation at the wall, Int. J. Thermal Sciences, 43 (2004), 531-539

[4] Huang C.-C Yan W. M. and Jang J.-H, Laminar mixed convection heat and mass transfer in a vertical rectangular ducts with film evaporation and condensation, Int. J. Heat Mass Transfer, 48 (2005), 1772-1784.

[5] Salah El-Din M. M., Fully developed forced convection in vertical channel with combined buoyancy forces, Int. Comm. Heat Mass Transfer, 19 (1992), 239-248.

[6] Fedorov A. G., R. Viskanta, A. A. Mohamed, R. R. Khaydarov., Turbulent heat and mass transfer in an asymmetrically heated, vertical parallel-plate channel, Int. J. Heat and Fluid Flow, 18 (1997), 307-315.

[7] Lee K. T., H. L. Tsai and W. M. Yan, Mixed convection heat and mass transfer in vertical rectangular ducts, Int. J. Heat and Mass Transfer, 40 (1997), 1621-1631.

[8] Yan W.M., Effects of film evaporation on laminar mixed convection heat and mass transfer in a vertical channel, Int. J. Heat Mass Transfer, 35 (1992), 34193429.

[9] Yan W.M. Mixed convection heat transfer in a vertical channel with film evaporation, Canadian J. Chemical Engineering, 71 (1993), 54-62

[10] Chow, L.C. and Chung, J.N, Evaporation of water into laminar stream of air and superheated steam, Int. J. Heat and Mass transfer, 26 (1983), 373-380.

[11]S. BenJabrallah, A. Belghith, J.P. CorriouConvective heat and mass transfer with evaporation of a falling film in a cavity Int J Therm Sci, 45 (2005), pp. 16-28. 